Introduction/Background* The Study 111 established LenPem as a treatment option in patients with advanced endometrial cancer following prior systemic therapy. LenPem has a higher objective response rate (ORR) and progression-free survival (PFS) with a different toxicity profile. The aim of this study was to gather data regarding the efficacy and safety of LenPem when used in the real-world treatment of EC.. This is the first study to examine LenPem using (?) in EC patients treated in Russia in real practice.

Methodology Retrospective chart review identified 34 patients administred LenPem for treatment of recurrent EC in Russia from March 2020 to March 2021. Demographic and clinical data were analized.

Result(s)* Thirty-four patients (median age 66,9 years, range 57-83 years; $20,6 \%$ of patients with serous carcinoma, $82,4 \%$ ECOG $\leq 1,64,7 \%$ pts with $\geq 2$ relapses, 38,2\% patients with $\geq 3$ prior platinum-based chemotherapeutic regimens) received 1-14 cycles (median 3). In 23 patients who were examined for efficacy, the response rate (RR) was $26.2 \%$ and stabizilazion observed in $69.6 \%$ cases. Incidence of grade $=>3$ adverse events was similar to that in prior studies (overall $97 \%)$. The most common side effects in this study and study 111 were fatigue $(64,7 \%$ and $51,1 \%$, respectively), hypertension $(47,1 \%$ and $61,7 \%$, respectively), diarrhea $(8,8 \%$ and $53,2 \%$ respectively) and stomatitis $(8,8 \%$ and $35,1 \%$ respectively). Overall, $29,4 \%$ pts required dose reduction due to toxicity and in $11,8 \%$ cases required discontinuation due to disease progression.

\begin{tabular}{lll}
\multicolumn{2}{l}{ Abstract 791 Table 1} \\
\hline & $\begin{array}{l}\text { Overall } \\
(\mathbf{n}=23), \%\end{array}$ & $\begin{array}{l}\text { Study 111 EC arm } \\
(\mathrm{n}=94), \%, \text { Inv. Assessment }\end{array}$ \\
\hline CR & 4,3 & 7,4 \\
PR & 21,7 & 29,8 \\
SD & 69,6 & 46,8 \\
PD & 4,3 & 10,6 \\
\hline
\end{tabular}

Conclusion* Outcomes of advanced EC patients treated in Russia with LenPem following prior systemic therapy are comparable to those demonstrated in the Study 111. LEN appears to be effective and safe in real world practice in EC.

\section{DEVELOPING NURSE LED PHONE CLINIC FOR ENDOMETRIAL CANCER FOLLOW-UP IN PRE COVID-19 ERA LEADING TO EFFICIENT FOLLOW UPS IN PANDEMIC TIMES}

${ }^{1} \mathrm{P}$ Bansal| ${ }^{*}{ }^{2} \mathrm{~B}$ Abdul. ${ }^{1}$ NN1 5BD, gynae oncology, UK; ${ }^{2}$ oxford university hospital, UK

\subsection{6/ijgc-2021-ESG0.198}

Introduction/Background* Systematic review says seventy percent of endometrial carcinoma recurrences are associated with symptoms. Is it safe to do nurse led phone clinic(NLPC) led by nurses and doctors.

Methodology Retrospective audit done on endometrial cancer patients in Northampton general hospital, treated from October 2013 and October 2018 and analysed the recurrence rate and presentation. Started NLPC in 2018. Wrote up a guideline and letter format for the nurses. Patient satisfaction Questionnaire done

Result(s)* Of 448 endometrial cancer patients in the above stated period, there were 57 recurrences. 8/57 patients were completely asymptomatic and was diagnosed in the first 2 years of follow up. However, 92\% of the patients presented with symptoms at the time of presentation.

On these databases NLPC was conducted with a prescribed format and on early stage endometrial cancers and further patient satisfaction assessed which was $98 \%$.

Conclusion* Majority of the cancer recurrence presented with symptoms, hence Nurse Led Phone follow up Clinics (NLPC) for selected cases is justified. Implementing NLPC leads to cut down in number clinic appointments, thus saving resources and give quality care to the more complex cases.

At the beginning of Covid 19 , phone clinics were simplified as practice was already in place in the department.

\section{IMPACT OF OBESITY ON SENTINEL LYMPH-NODE MAPPING IN PATIENTS WITH APPARENT EARLY-STAGE ENDOMETRIAL CANCER: A PROPENSITY-MATCHED MULTICENTER STUDY}

${ }^{1} V$ Vargiu*, ${ }^{2} \mathrm{~A}$ Rosati, ${ }^{3}$ VA Capozzi, ${ }^{4} \mathrm{G}$ Sozzi, ${ }^{3} \mathrm{R}$ Berretta, ${ }^{4} \mathrm{~V}$ Chiantera, ${ }^{2} \mathrm{G}$ Scambia ${ }^{2} \mathrm{~F}$ Fanfani, ${ }^{1} \mathrm{~F}$ Cosentino. ' Gemelli Molise SpA, Department of Gynecologic Oncology, Campobasso, Italy; ${ }^{2}$ Fondazione Policlinico Universitario A. Gemelli IRCCS, Department of Women's and Children's Health, Rome, Italy; ' University of Parma, Department of Gynecology and Obstetrics, Parma, Italy; ${ }^{4}$ ARNAS Civico Di Cristina Benfratelli, Department of Gynecologic Oncology, Palermo, Italy

\subsection{6/ijgc-2021-ESG0.199}

Introduction/Background* Obese patients pose both surgical and anesthetic challenges, as their comorbidities contribute to adverse outcomes.

In this setting, minimally invasive approach and the introduction of the Sentinel Lymph-Node (SLN) algorithm in endometrial cancer (EC) treatment acquire a particular relevance, allowing to reduce both operative times and surgical difficulties.

However, conflicting data exists on the impact of Body Mass Index (BMI) on SLN detection.

The primary study endpoint was to investigate the impact of obesity on overall detection rate, bilateral mapping, and mapping failure rate. In addition, we evaluated possible differences in terms of surgical management and 'empty packet dissection' rate among the two study groups.

Methodology Multicenter, propensity-matched, retrospective study.

Data of patients with apparently early-stage EC were retrospectively retrieved. Study population was divided into women with BMI $</ \geq 30$ (respectively Group-1 and Group-2). To lower the selection bias, a propensity matched analysis was performed. Matching was based on the most relevant variables impacting SLN detection, such as histotype (endometrioid vs non-endometrioid), age $(</ \geq 65$ years old), presence of lymphvascular space invasion.

Result(s)* Eight-hundred forty-four women were enrolled in the study. After a 1:1 propensity matched analysis, a total of 764 patients were identified (Group-1 n=382, Group-2 $\mathrm{n}=382$ ). A 1.156-fold increase in the risk of mapping failure for every 5 units of increase in BMI (OR 1.156, 95\% CI 1.033-1.294, $\mathrm{p}=0.012$ ) was found, with a consequently decrease in bilateral mapping and overall detection rate 


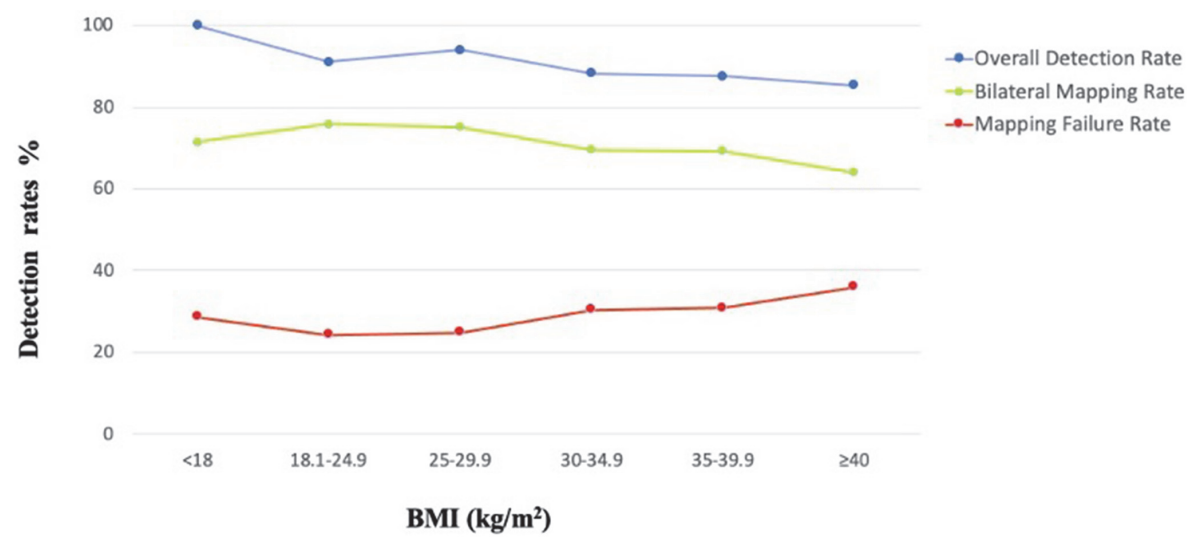

Overall detection rate: $\mathrm{OR} 0.785,95 \% \mathrm{CI} 0.670-0.920, \mathrm{p}=0.003$

Successful bilateral mapping: OR $0.865,95 \%$ CI $0.773-0.968, p=0.012$

Mapping failure risk: OR 1.156, 95\% CI 1.033-1.294, $\mathrm{p}=0.012$

Abstract 806 Figure 1 SLN detection rates per 5-unit of BMI increase and bionominal logistic regresion analysis in the propensity matched population

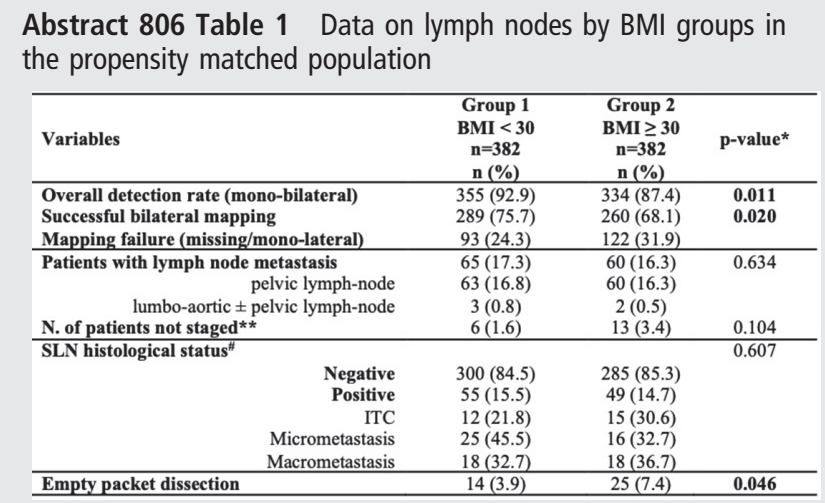

(respectively OR $0.865,95 \%$ CI $0.773-0.968, \mathrm{p}=0.012$ and OR 0.785 , 95\% CI $0.670-0.920, \mathrm{p}=0.003$ ) (figure 1). Furthermore, in the group of obese patients, in 25 cases $(7.4 \%)$ the SLN dissection did not lead to the identification of lymphnode tissue at final histopathological examination ('empty packet dissection') (Group1 vs Group 2: $3.9 \%$ vs $7.4 \%$, $\mathrm{p}=0.046$ ) (table 1).

Conclusion* Controlling for the variables that have been proved to negatively influence the SLN detection rate, BMI was confirmed as a statistically relevant predictor of mapping failure. In addition, obese women had a significantly higher odds of empty packet dissection, which could lead to an unintended surgical understaging.

\section{FERTILITY PRESERVATION IN ENDOMETRIAL CANCER: PERINATAL AND ONCOLOGIC OUTCOMES}

V García Pineda*, M Turiel, M Gracia, I Zapardiel, MD Diestro, J Siegrist, Y Pérez Martínez, A Hernández. La Paz University Hospital, Gynaecological Oncology, Madrid, Spain

\subsection{6/ijgc-2021-ESGO.200}

Introduction/Background* The aim of our study is to evaluate the oncological and perinatal outcomes in young women diagnosed with atypical endometrial hyperplasia (AEH) or endometrial cancer (EC) treated with hormone therapy.
Methodology A single institutional ambispective study was performed including all patients diagnosed with $\mathrm{AEH}$ or $\mathrm{EC}$ grade 1 without myometrial invasion who received hormone therapy between January 2011 and July 2021. We analyzed the complete response rate and recurrence rate of disease and pregnancy rate in these patients as well as perinatal results (live births rate, type of delivery and perinatal morbidity). In addition, we evaluated complete response rate according to type of hormone therapy, dosage received and treatment length.

A review of literature was performed to identify studies involving patients with $\mathrm{AEH}$ or $\mathrm{EC}$ who received fertility sparing management.

All statistical analysis were performed using the software SPSS Statistics v.24.0 (IBM Corp., Armonk, NY, USA).

Result(s)* The results after final statistical analysis will be available when we complete the collection of follow-up data for conservatively treated patients.

Conclusion* Conservative management with progestins of young patients with AEH or EC grade 1 limited to the endometrium is an acceptable possibility given the high remission rate and live birth rates achieved. However, definitive surgical treatment cannot be avoided given the high recurrence rate described in the literature.

\section{A RARE SITUATION: HERNIATION OF BOWEL THROUGH 5 MM TROCAR SITE IN A PATIENT WITH ENDOMETRIAL CARCINOMA}

NA Vural ${ }^{*}$, F Vaizoğlu, S Soylu, B Erdem, N Çetinkaya Kocadal. Başakşehir Çam ve Sakura Şehir Hastanesi, Turkey

\subsection{6/ijgc-2021-ESGO.201}

Introduction/Background* Hernia formation through $5 \mathrm{~mm}$-trocar-site has incidence between $1-6 \%$ in the literature. Practically fascial defect at the 10 -mm-trocar site is closed, while suturing at the 5 -mm-trocar site is not preferred. Because of its rarity, a possible hernia can be underestimated by the clinician and severe intestinal damage may occure as complication of minimally invasive surgery. Here we present a case -who had been operated via laparoscopy due to endometrial 